\title{
Combinatorial Game Distributions of Steiner Systems
}

\author{
Yuki Irie* \\ Research Alliance Center for Mathematical Sciences \\ Tohoku University \\ Miyagi, Japan \\ yirie@tohoku.ac.jp
}

Submitted: Dec 31, 2019; Accepted: Nov 24, 2021; Published: Dec 17, 2021

(C) The author. Released under the CC BY license (International 4.0).

\begin{abstract}
The $\mathscr{P}$-position sets of some combinatorial games have special combinatorial structures. For example, the $\mathscr{P}$-position set of the hexad game, first investigated by Conway and Ryba, is the block set of the Steiner system $S(5,6,12)$ in the shuffle numbering, denoted by $\mathcal{D}_{\mathrm{sh}}$. However, few games were known to be related to Steiner systems in this way. For a given Steiner system, we construct a game whose $\mathscr{P}$-position set is its block set. By using constructed games, we obtain the following two results. First, we characterize $\mathcal{D}_{\text {sh }}$ among the 5040 isomorphic $S(5,6,12)$ with point set $\{0,1, \ldots, 11\}$. For each $S(5,6,12)$, our construction produces a game whose $\mathscr{P}$-position set is its block set. From $\mathcal{D}_{\text {sh }}$, we obtain the hexad game, and this game is characterized as the unique game with the minimum number of positions among the obtained 5040 games. Second, we characterize projective Steiner triple systems by using game distributions. Here, the game distribution of a Steiner system $\mathcal{D}$ is the frequency distribution of the numbers of positions in games obtained from Steiner systems isomorphic to $\mathcal{D}$. We find that the game distribution of an $S(t, t+1, v)$ can be decomposed into symmetric components and that a Steiner triple system is projective if and only if its game distribution has a unique symmetric component.
\end{abstract}

Mathematics Subject Classifications: 05B05, 91A46

*This work was partially supported by JSPS KAKENHI Grant Number JP20K14277. The paper is a substantially revised version of Section 2.A of the author's Ph.D. thesis, which was prepared under the supervision of Masaaki Kitazume for Chiba University. This revision includes adding a result that characterizes projective Steiner triple systems. 


\section{Introduction}

The starting point of this paper is a two-player game called the hexad game ${ }^{1}[6,13]$. This game is played with six coins and a strip of cells indexed by [12], where [12] = $\{0,1, \ldots, 11\}$. The six coins are placed on distinct cells indexed by $p_{1}, p_{2}, \ldots, p_{6}$ with $\sum p_{i} \geqslant 21$. Two players alternately move a coin to a smaller empty cell with the restriction that the sum of indices of the six non-empty cells is at least 21. A player who cannot move loses. Figure 1 shows an example of the hexad game. Note that a position in the hexad game can be represented as an element of $\left(\begin{array}{c}{[12]} \\ 6\end{array}\right)$, where $\left(\begin{array}{c}{[12]} \\ 6\end{array}\right)=\{P \subset[12]:|P|=6\}$.

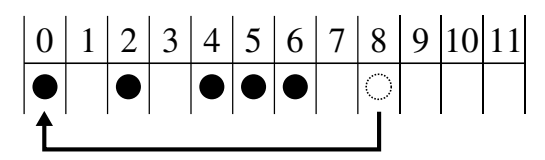

The first player moves 8 to 0 .



The second player moves 6 to 1 and wins.

Figure 1: An example of the hexad game with six coins initially placed on 2, 4, 5, 6, 8, and 9 .

The hexad game is notable because its $\mathscr{P}$-position set is the block set of a Steiner system $S(5,6,12)[6,13]$. Here, a $\mathscr{P}$-position is a winning position for the Previous player (i.e., the second player to move) $;^{2}$ a pair $([v], \mathcal{B})$ is called a Steiner system $S(t, k, v)$ with point set $[v]$ and block set $\mathcal{B}$ if $\mathcal{B} \subseteq\left(\begin{array}{c}{[v]} \\ k\end{array}\right)$ and every $T \in\left(\begin{array}{c}{[v]} \\ t\end{array}\right)$ is contained in a unique $B \in \mathcal{B}$. Further, Conway and Sloane [6] found a game whose $\mathscr{P}$-position set is the block set of a Steiner system $S(5,8,24)$. No general methods, however, were known to find such games related to Steiner systems as the above games.

For a given Steiner system, we construct a game whose $\mathscr{P}$-position set is equal to its block set (Corollary 10). ${ }^{3}$ We here illustrate our construction using the $S(1,2,4)$ with block set $\{\{0,2\},\{1,3\}\}$; our aim is to obtain the game shown in Figure 2. We use a digraph to represent a game. Let $\Gamma$ be the digraph with vertex set $\left(\begin{array}{c}{[4]} \\ 2\end{array}\right)$ and edge set

$$
\left\{\left(P, P^{(p q)}\right) \in\left(\begin{array}{c}
{[4]} \\
2
\end{array}\right)^{2}: q<p, p \in P, q \notin P\right\},
$$

where $(p q)$ is the transposition of $p$ and $q$, that is, $P^{(p q)}=P \cup\{q\} \backslash\{p\}$ when $p \in P$ and $q \notin P$. We denote by $\mathcal{P}_{\Gamma}$ and $\mathcal{E}_{\Gamma}$ the vertex and edge sets of $\Gamma$, respectively. For example, $\left(\{2,3\},\{2,3\}^{(30)}\right)=(\{2,3\},\{0,2\}) \in \mathcal{E}_{\Gamma}$. For $P \in \mathcal{P}_{\Gamma}$, let $\mathcal{J}_{\Gamma}(P)$ and $\mathcal{O}_{\Gamma}(P)$ be the sets of in-neighbors and out-neighbors of $P$, respectively. That is,

$$
\mathcal{J}_{\Gamma}(P)=\left\{R \in \mathcal{P}_{\Gamma}:(R, P) \in \mathcal{E}_{\Gamma}\right\} \quad \text { and } \quad \mathcal{O}_{\Gamma}(P)=\left\{Q \in \mathcal{P}_{\Gamma}:(P, Q) \in \mathcal{E}_{\Gamma}\right\} .
$$

\footnotetext{
${ }^{1}$ According to [13], the hexad game was first investigated by Conway and Ryba.

${ }^{2}$ The $\mathscr{P}$-position set of the hexad game is a subset of $\left(\begin{array}{c}{[12]} \\ 6\end{array}\right)$. For example, a position $P \in\left(\begin{array}{c}{[12]} \\ 6\end{array}\right)$ with $\sum_{p \in P} p=21$ is a $\mathscr{P}$-position in the hexad game.

${ }^{3}$ Since we start with a $\mathscr{P}$-position set, our approach may be referred to as reverse game theory or mechanism design (see [12]).
} 
For example, $\mathcal{J}_{\Gamma}(\{0,2\})=\{\{1,2\},\{0,3\},\{2,3\}\}$. Define

$$
\mathcal{P}_{\Gamma}^{\mathcal{B}}=\mathcal{B} \cup \bigcup_{B \in \mathcal{B}} \mathcal{J}_{\Gamma}(B)
$$

where $\mathcal{B}=\{\{0,2\},\{1,3\}\}$. Then $\mathcal{P}_{\Gamma}^{\mathcal{B}}=\left(\begin{array}{c}{[4]} \\ 2\end{array}\right) \backslash\{\{0,1\}\}$. Let $\operatorname{Ind}\left(\Gamma, \mathcal{P}_{\Gamma}^{\mathcal{B}}\right)$ denote the subgraph induced in $\Gamma$ by $\mathcal{P}_{\Gamma}^{\mathcal{B}}$, that is, the digraph with vertex set $\mathcal{P}_{\Gamma}^{\mathcal{B}}$ and edge set

$$
\left\{(P, Q) \in \mathcal{E}_{\Gamma}: P, Q \in \mathcal{P}_{\Gamma}^{\mathcal{B}}\right\} .
$$

We consider the digraph $\operatorname{Ind}\left(\Gamma, \mathcal{P}_{\Gamma}^{\mathcal{B}}\right)$ as the following two-player game. Before a game begins, we put a token on a starting vertex $P \in \mathcal{P}_{\Gamma}^{\mathcal{B}}$. The first player moves the token from $P$ to an out-neighbor $Q$ of $P$. Similarly, the second player moves it from $Q$ to an outneighbor $R$ of $Q$. In this way, the two players alternately move the token from the current vertex to one of its out-neighbors. A player who cannot move loses. In this manner, we can view a digraph as a two-player game. Then the $\mathscr{P}$-position set of $\operatorname{Ind}\left(\Gamma, \mathcal{P}_{\Gamma}^{\mathcal{B}}\right)$ is $\mathcal{B}$.

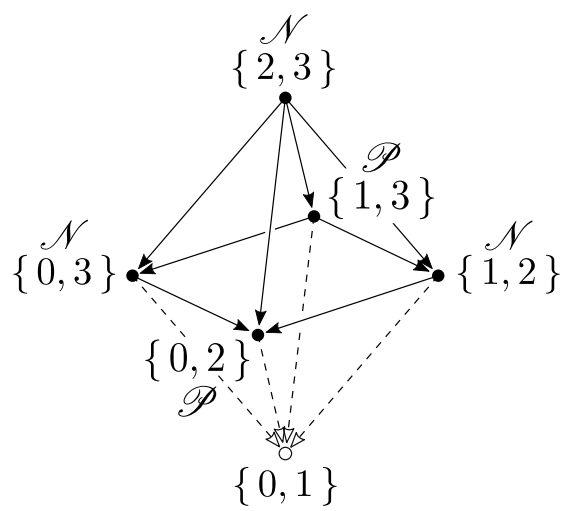

Figure 2: A game whose $\mathscr{P}$-position set is $\{\{0,2\},\{1,3\}\}$. The labels $\mathscr{P}$ and $\mathscr{N}$ indicate winning positions for the previous and next players, respectively.

For example, if $\{1,3\}$ is the starting vertex, then the first player can move the token only to $\{0,3\}$ or $\{1,2\}$, so the second player can move it to $\{0,2\}$ and win. Thus $\{1,3\}$ is a winning position for the previous player, that is, a $\mathscr{P}$-position. In the same way, for a given Steiner system, we can construct a game whose $\mathscr{P}$-position set is its block set (Corollary 10).

In this paper, we investigate the frequency distribution of the numbers of positions of games obtained from a Steiner system. Let $\mathcal{D}$ be an $S(t, k, v)$ with point set $[v]$, and let $N$ be the index of the automorphism group of $\mathcal{D}$ in the symmetric group $\operatorname{Sym}([v])$ on $[v]$. Then there are $N$ Steiner systems $S(t, k, v)$ with point set $[v]$ isomorphic to $\mathcal{D}$, and, for each of them, we can construct a game whose $\mathscr{P}$-position set is its block set. The frequency distribution of the numbers of positions (vertices) of the obtained $N$ games is called the game distribution of $\mathcal{D}$. For example, there are three $S(1,2,4)$ with point set [4], and their block sets are as follows:

$$
\mathcal{B}=\{\{0,2\},\{1,3\}\}, \quad \mathcal{B}^{\prime}=\{\{0,1\},\{2,3\}\}, \quad \mathcal{B}^{\prime \prime}=\{\{0,3\},\{1,2\}\} .
$$


As we have seen, $\left|\mathcal{P}_{\Gamma}^{\mathcal{B}}\right|=5$. A direct computation shows that

$$
\left|\mathcal{P}_{\Gamma}^{\mathcal{B}^{\prime}}\right|=\left|\left(\begin{array}{c}
{[4]} \\
2
\end{array}\right)\right|=6 \quad \text { and } \quad\left|\mathcal{P}_{\Gamma}^{\mathcal{B}^{\prime \prime}}\right|=\left|\left(\begin{array}{c}
{[4]} \\
2
\end{array}\right) \backslash\{\{0,1\},\{0,2\}\}\right|=4 .
$$

Therefore the game distribution of an $S(1,2,4)$ is as follows:

$$
\begin{array}{c|ccc|c}
\text { \# positions } & 4 & 5 & 6 & \text { Total } \\
\hline \text { \# games } & 1 & 1 & 1 & 3
\end{array}
$$

By using game distributions, we present the following two results.

First, we characterize the Steiner system $S(5,6,12)$ in the shuffle numbering, denoted by $\mathcal{D}_{\mathrm{sh}}$. It is well-known that $S(5,6,12)$ is unique up to isomorphism and its automorphism group is the sporadic simple Mathieu group $M_{12}[17,18]$. Since the index of $M_{12}$ in $\operatorname{Sym}([12])$ equals 5040 , it follows that there are $5040 S(5,6,12)$ with point set [12], and our construction produces 5040 games. Here, $\mathcal{D}_{\mathrm{sh}}$ is one of the $5040 S(5,6,12)$. The name of the shuffle numbering comes from the fact that the automorphism group of $\mathcal{D}_{\text {sh }}$ is generated by the two permutations $i \mapsto 11-i$ and $i \mapsto \min (2 i, 23-2 i)$ of [12], called the Mongean shuffle, and $\mathcal{D}_{\mathrm{sh}}$ has many interesting properties $[7,10]$. In fact, the $\mathscr{P}$ position set of the hexad game is the block set of $\mathcal{D}_{\mathrm{sh}}[6,13]$. Conversely, our construction produces the hexad game from $\mathcal{D}_{\mathrm{sh}}$. Surprisingly, this game can be characterized as the unique game with the minimum number of positions among the obtained 5040 games; this result is easily seen by using the game distribution of an $S(5,6,12)$ (Theorem 16). In particular, we can characterize $\mathcal{D}_{\text {sh }}$ by using games.

Second, we characterize projective Steiner triple systems among all Steiner triple systems. In this paragraph, we consider non-isomorphic designs unlike in the previous paragraph. The projective geometry $\mathrm{PG}(d, 2)$ forms a Steiner system $S\left(2,3,2^{d+1}-1\right)$, called the projective Steiner triple system of order $2^{d+1}-1$ (Example 2). It is known that there are 80 non-isomorphic Steiner systems $S(2,3,15)$ [4], and one of them is projective. How can we characterize the projective one among them? In combinatorial design theory, characterizations of particular designs, including projective Steiner triple systems, have been studied (see, for example, $[1,3,9,11,16])$. In this paper, we show that the game distribution of an $S(t, t+1, v)$ can be decomposed into symmetric components (Proposition 19). We then prove that an $S(2,3, v)$ is projective if and only if its game distribution has a unique symmetric component (Theorem 23).

This paper is organized as follows. In Section 2, we recall the concepts of a Steiner system and an impartial game. Section 3 contains the construction of games whose $\mathscr{P}_{-}$ position set equals the block set of a Steiner system. In Section 4, we introduce game distributions and then characterize the $S(5,6,12)$ in the shuffle numbering. In Section 5 , it is shown that the game distribution of an $S(t, t+1, v)$ can be decomposed into symmetric components. We finally characterize projective Steiner triple systems by using game distributions in Section 6.

\section{Preliminaries}

We recall some facts about Steiner systems and impartial games that will be used later. 


\subsection{Steiner Systems}

Let $t$ be a nonnegative integer and let $v, k$, and $\lambda$ be positive integers with $t \leqslant k \leqslant v$. A $t-(v, k, \lambda)$ design $\mathcal{D}$ is a pair $\left(\mathcal{V}_{\mathcal{D}}, \mathcal{B}_{\mathcal{D}}\right)$ where $\mathcal{V}_{\mathcal{D}}$ is a set with $\left|\mathcal{V}_{\mathcal{D}}\right|=v$ and $\mathcal{B}_{\mathcal{D}}$, called the block set of $\mathcal{D}$, is a subset of $\left(\begin{array}{c}V_{\mathcal{D}} \\ k\end{array}\right)$ such that every $T \in\left(\begin{array}{c}\mathcal{V}_{\mathcal{D}} \\ t\end{array}\right)$ is contained in exactly $\lambda$ blocks. Note that a $t-(v, k, 1)$ design is a Steiner system $S(t, k, v)$. Throughout this paper, we assume that $\mathcal{V}_{\mathcal{D}}=[v]=\{0,1, \ldots, v-1\}$ unless otherwise stated. If $\mathcal{D}$ is a $t-(v, k, \lambda)$ design, then it is also an $i-\left(v, k, \lambda_{i}\right)$ design for $0 \leqslant i \leqslant t$, where

$$
\lambda_{i}=\frac{\left(\begin{array}{c}
v-i \\
t-i
\end{array}\right)}{\left(\begin{array}{c}
k-i \\
t-i
\end{array}\right)} \lambda .
$$

Note that $\lambda_{0}$ equals the number of blocks of $\mathcal{D}$.

Example 1. Let $\mathcal{B}=\{\{0,1\},\{2,3\}, \ldots,\{2 v-2,2 v-1\}\}$. Then $([2 v], \mathcal{B})$ is an $S(1,2,2 v)$.

Example 2 (Projective Steiner triple systems). Let $\mathbb{F}_{2}$ be the finite field with two elements. Let

$$
\mathcal{V}=\mathbb{F}_{2}^{d+1} \backslash\{(0, \ldots, 0)\} \quad \text { and } \quad \mathcal{B}=\left\{\{p, q, p+q\} \in\left(\begin{array}{l}
\mathcal{V} \\
3
\end{array}\right):\{p, q\} \in\left(\begin{array}{l}
\mathcal{V} \\
2
\end{array}\right)\right\} .
$$

We see that $(\mathcal{V}, \mathcal{B})$ is a Steiner system $S\left(2,3,2^{d+1}-1\right)$. This design is called the projective Steiner triple system of order $2^{d+1}-1$, since $\mathcal{V}$ and $\mathcal{B}$ can be regarded as the point and line sets of the projective geometry $\mathrm{PG}(d, 2)$. For example, if $d=2$, then the blocks are as shown in Figure 3.

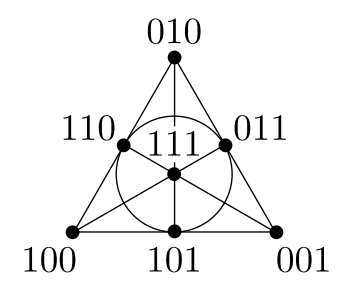

Figure 3: The blocks of the projective Steiner triple system of order 7. For example, $\{(1,0,0),(1,1,0),(0,1,0)\}$ and $\{(1,1,0),(1,0,1),(0,1,1)\}$ are blocks.

We next define isomorphisms. Let $\mathcal{D}$ and $\mathcal{D}^{\prime}$ be $t-(v, k, \lambda)$ designs. A bijection $\phi: \mathcal{V}_{\mathcal{D}} \rightarrow \mathcal{V}_{\mathcal{D}^{\prime}}$ is called an isomorphism if $\phi(B) \in \mathcal{B}_{\mathcal{D}^{\prime}}$ for every $B \in \mathcal{B}_{\mathcal{D}}$, where $\phi(B)=$ $\{\phi(b): b \in B\}$. If there is an isomorphism between $\mathcal{D}$ and $\mathcal{D}^{\prime}$, then they are said to be isomorphic. An isomorphism from $\mathcal{D}$ to itself is called an automorphism of $\mathcal{D}$. Let $\operatorname{Aut}(\mathcal{D})$ denote the group of automorphisms of $\mathcal{D}$.

Example 3. Let $\mathcal{D}$ be the $S(1,2,4)$ with block set $\{\{0,1\},\{2,3\}\}$. The automorphism group of $\mathcal{D}$ is generated by the two permutations $\left(\begin{array}{lll}0 & 1\end{array}\right)$ and $\left(\begin{array}{lll}0 & 2\end{array}\right)\left(\begin{array}{ll}1 & 3\end{array}\right)$. This group is isomorphic to the dihedral group $D_{8}$ of order 8 . It is easy to see that $S(1,2,4)$ is unique up to isomorphism. In general, $S(1,2,2 v)$ is unique up to isomorphism. 
Remark 4. A Steiner system $S(2,3, v)$ is called a Steiner triple system (see [3] for details). Kirkman [14] showed that an $S(2,3, v)$ exists if and only if $v \equiv 1,3(\bmod 6)$. Although $S(2,3,7)$ and $S(2,3,9)$ are unique up to isomorphism, $S(2,3, v)$ is not unique for $v>$ $9[8,15]$. For example, there are 80 non-isomorphic $S(2,3,15)[4]$ and one of them is projective. Let $\mathcal{D}$ be an $S(2,3, v)$. By the Veblen-Young theorem [16], the following two conditions are equivalent:

(1) $\mathcal{D}$ is projective, that is, it is isomorphic to a projective Steiner triple system.

(2) If $\{a, b, c\},\{a, d, e\}$, and $\{b, d, f\}$ are distinct three blocks of $\mathcal{D}$, then $\{c, e, f\}$ is also a block (see Figure 4 ).

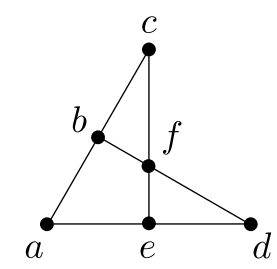

Figure 4: A characterization of projective Steiner triple systems.

\subsection{Impartial Games}

We recall the concept of an impartial game (see, for example, $[2,5]$ for more details).

We represent a game as a digraph. Let $\Gamma$ be a digraph $\left(\mathcal{P}_{\Gamma}, \mathcal{E}_{\Gamma}\right)$ satisfying

$$
\lg _{\Gamma}(P)<\infty \text { for every } P \in \mathcal{P}_{\Gamma}
$$

where $\lg _{\Gamma}(P)$ is the maximum length of a walk from $P$. The vertex set $\mathcal{P}_{\Gamma}$ is called the position set of $\Gamma$. If $(P, Q) \in \mathcal{E}_{\Gamma}$, then $Q$ is called an option of $P$. A position without options is called a terminal position. Suppose that $\Gamma$ has at least one position; then $\Gamma$ has a terminal position. We consider $\Gamma$ as a two-player game in the way described in the introduction. This kind of game is called an impartial game, and every (short) impartial game can be represented in this manner. Thus we call a digraph satisfying (2.2) simply a game.

Example 5. Let

$$
\Gamma^{1}=([4],\{(3,2),(3,1),(2,1),(2,0),(1,0)\}) \quad \text { and } \quad \Gamma^{2}=([2],\{(1,0),(0,1)\}) .
$$

See Figure 5. Then $\Gamma^{1}$ is a game and position 0 is a terminal position in $\Gamma^{1}$. However, $\Gamma^{2}$ is not a game since the walk $(1,0,1,0, \ldots)$ has infinite length.

Example 6 (Welter's game and the hexad game). Let $\Gamma_{v, k}$ be the game with position set $\left(\begin{array}{c}{[v]} \\ k\end{array}\right)$ and edge set

$$
\left\{\left(P, P^{(p q)}\right) \in\left(\begin{array}{c}
{[v]} \\
k
\end{array}\right)^{2}: q<p, p \in P, q \notin P\right\}
$$



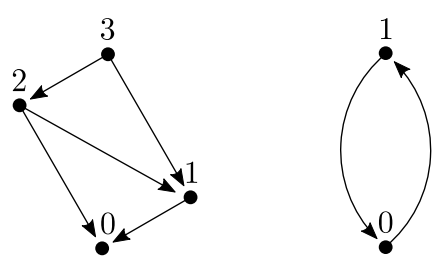

Figure 5: $\Gamma^{1}$ and $\Gamma^{2}$.

The game $\Gamma_{v, k}$ is called Welter's game. Let

$$
\mathcal{P}_{\mathrm{Hxd}}=\left\{P \in\left(\begin{array}{c}
{[12]} \\
6
\end{array}\right): \sum_{p \in P} p \geqslant 21\right\} .
$$

The subgraph $\operatorname{Ind}\left(\Gamma_{12,6}, \mathcal{P}_{\mathrm{Hxd}}\right)$ induced in $\Gamma_{12,6}$ by $\mathcal{P}_{\mathrm{Hxd}}$ is called the hexad game and is denoted by Hxd.

We next define $\mathscr{P}$-position sets. Let $\mathscr{P}$ and $\mathscr{N}$ be two symbols. For a game $\Gamma$ and a position $P \in \mathcal{P}_{\Gamma}$, the outcome $o_{\Gamma}(P)$ of $P$ is defined recursively by

$$
o_{\Gamma}(P)= \begin{cases}\mathscr{N} & \text { if } P \text { has an option } Q \text { with } o_{\Gamma}(Q)=\mathscr{P} \\ \mathscr{P} & \text { otherwise. }\end{cases}
$$

Recall that $\Gamma$ has a terminal position if it has at least one position. By definition, the outcome of a terminal position is $\mathscr{P}$. An easy induction shows that the previous player can force a win if and only if the outcome of the starting position is $\mathscr{P}$. Let

$$
\mathcal{W}_{\Gamma}=\left\{P \in \mathcal{P}_{\Gamma}: o_{\Gamma}(P)=\mathscr{P}\right\} .
$$


$\mathcal{W}_{\Gamma}$ is an independent set in $\Gamma$, that is, $(B, C) \notin \mathcal{E}_{\Gamma}$ for any $B, C \in \mathcal{W}_{\Gamma}$. As we will see in the next section, our construction, described in the introduction, can be applied to an independent set in $\Gamma$. Note that if $\mathcal{B}$ is the block set of an $S(t, k, v)$ with $t<k$, then $\mathcal{B}$ is an independent set in $\Gamma_{v, k}$.

Example 7. Let $\Gamma^{1}$ be as in Example 5. The outcomes of the four positions are shown in Figure 6. For example, the previous player can force a win if we start at position 3.

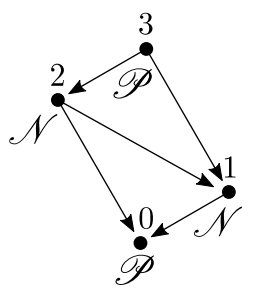

Figure 6: Outcomes of positions. 
Remark 8. Let $\mathcal{D}_{\mathrm{sh}}=\left([12], \mathcal{W}_{\mathrm{Hxd}}\right)$. Then $\mathcal{D}_{\mathrm{sh}}$ is an $S(5,6,12)$, called the Steiner system $S(5,6,12)$ in the shuffle numbering $[6,13]$. For example, if $P$ is an element in $\left(\begin{array}{c}{[12]} \\ 6\end{array}\right)$ with $\sum_{p \in P} p=21$, then it is a terminal position in $\mathrm{Hxd}$, so $P \in \mathcal{W}_{\mathrm{Hxd}}$. Incidentally, the set $\mathcal{W}_{\text {Hxd }}$ can be obtained as the orbit

$$
\left\{\{0,1,2,3,4,11\}^{\pi}: \pi \in G\right\},
$$

where $\{0,1,2,3,4,11\}^{\pi}=\left\{0^{\pi}, 1^{\pi}, 2^{\pi}, 3^{\pi}, 4^{\pi}, 11^{\pi}\right\}$ and $G$ is the group generated by the two permutations $i \mapsto 11-i$ and $i \mapsto \min (2 i, 23-2 i)$ of [12], which is the automorphism group of $\mathcal{D}_{\mathrm{sh}}$. We can also explicitly describe $\mathcal{W}_{\mathrm{Hxd}}$ by using the following $3 \times 4$ array, called the MINIMOG in the shuffle numbering (see [7]).

\begin{tabular}{ccccc|} 
o & 6 & 3 & 0 & 9 \\
+ & 5 & 2 & 7 & 10 \\
- & 4 & 1 & 8 & 11 \\
\hline
\end{tabular}

\section{Construction of Games}

For an independent set $\mathcal{B}$ in a game $\Gamma$, we construct a game whose $\mathscr{P}$-position set equals $\mathcal{B}$. In particular, our construction works for the block set $\mathcal{B}$ of an $S(t, k, v)$ with $t<k$ since $\mathcal{B}$ is an independent set in $\Gamma_{v, k}$.

For an independent set $\mathcal{B}$ in a game $\Gamma$, define $\mathcal{P}_{\Gamma}^{\mathcal{B}}$ by $(1.1)$, that is, $\mathcal{P}_{\Gamma}^{\mathcal{B}}=\mathcal{B} \cup \bigcup_{B \in \mathcal{B}} \mathcal{J}_{\Gamma}(B)$. When no confusion can arise, we write $\mathcal{P}^{\mathcal{B}}, \mathcal{J}(P)$, and $\mathcal{O}(P)$ instead of $\mathcal{P}_{\Gamma}^{\mathcal{B}}, \mathcal{J}_{\Gamma}(P)$, and $\mathcal{O}_{\Gamma}(P)$, respectively. Let $\Phi(\Gamma, \mathcal{B})$ denote the family of the position sets of induced subgraphs of $\Gamma$ whose $\mathscr{P}$-position sets equal $\mathcal{B}$, that is,

$$
\Phi(\Gamma, \mathcal{B})=\left\{\mathcal{Q} \subseteq \mathcal{P}_{\Gamma}: \mathcal{W}_{\operatorname{Ind}(\Gamma, \mathcal{Q})}=\mathcal{B}\right\}
$$

Theorem 9. If $\Gamma$ is a game and $\mathcal{B}$ is an independent set in $\Gamma$, then

$$
\Phi(\Gamma, \mathcal{B})=\left\{\mathcal{Q}: \mathcal{B} \subseteq \mathcal{Q} \subseteq \mathcal{P}^{\mathcal{B}}\right\}
$$

in particular, $\mathcal{W}_{\operatorname{Ind}\left(\Gamma, \mathcal{P}^{\mathcal{B}}\right)}=\mathcal{B}$ and $\mathcal{P}^{\mathcal{B}}$ is the maximum element of $\Phi(\Gamma, \mathcal{B})$ with respect to inclusion.

Proof. We begin by showing that if $\mathcal{B} \subseteq \mathcal{Q} \subseteq \mathcal{P}^{\mathcal{B}}$, then $\mathcal{Q} \in \Phi(\Gamma, \mathcal{B})$. Let $\Delta=\operatorname{Ind}(\Gamma, \mathcal{Q})$. It suffices to show that $\mathcal{W}_{\Delta}=\mathcal{B}$. Let $Q \in \mathcal{Q}$ and $l=\lg _{\Delta}(Q)$. We show that $Q \in \mathcal{W}_{\Delta}$ if and only $Q \in \mathcal{B}$ by induction on $l$. If $l=0$, then $Q \in \mathcal{W}_{\Delta}$ and $Q \in \mathcal{B}$ since $Q$ is a terminal position in $\Delta$ and $Q \in \mathcal{Q} \subseteq \mathcal{P}^{\mathcal{B}}$. Suppose that $l>0$. First, we show that if $Q \in \mathcal{B}$, then $Q \in \mathcal{W}_{\Delta}$. Let $R$ be an option of $Q$. Since $\mathcal{B}$ is an independent set, it follows that $R \notin \mathcal{B}$. By the induction hypothesis, $R \notin \mathcal{W}_{\Delta}$. This implies that $Q \in \mathcal{W}_{\Delta}$. Next, we show that if $Q \notin \mathcal{B}$, then $Q \notin \mathcal{W}_{\Delta}$. Since $Q \in \mathcal{Q} \subseteq \mathcal{P}^{\mathcal{B}}$, it follows that $Q$ has an option $B$ with $B \in \mathcal{B}$. By the induction hypothesis, $B \in \mathcal{W}_{\Delta}$, so $Q \notin \mathcal{W}_{\Delta}$. Therefore $\mathcal{W}_{\Delta}=\mathcal{B}$.

It remains to prove that if $\mathcal{Q} \in \Phi(\Gamma, \mathcal{B})$, then $\mathcal{B} \subseteq \mathcal{Q} \subseteq \mathcal{P}^{\mathcal{B}}$. It is obvious that $\mathcal{Q} \supseteq \mathcal{B}$. We show that $\mathcal{Q} \subseteq \mathcal{P}^{\mathcal{B}}$. Let $Q \in \mathcal{Q}$ and $\Delta=\operatorname{Ind}(\Gamma, \mathcal{Q})$. If $Q \in \mathcal{B}$, then $Q \in \mathcal{P}^{\mathcal{B}}$. Suppose that $Q \notin \mathcal{B}$. Since $\mathcal{B}=\mathcal{W}_{\Delta}$, it follows that $Q \notin \mathcal{W}_{\Delta}$, and that $Q$ has an option $B$ with $B \in \mathcal{W}_{\Delta}=\mathcal{B}$. This implies that $Q \in \mathcal{J}(B) \subseteq \mathcal{P}^{\mathcal{B}}$. Therefore $\mathcal{Q} \subseteq \mathcal{P}^{\mathcal{B}}$. 
For a $t$ - $(v, k, \lambda)$ design $\mathcal{D}$, let $\Gamma^{\mathcal{D}}$ denote the game $\operatorname{Ind}\left(\Gamma_{v, k}, \mathcal{P}^{\mathcal{D}}\right)$, where $\mathcal{P}^{\mathcal{D}}=\mathcal{P}^{\mathcal{B}_{\mathcal{D}}}$. If $\mathcal{B}_{\mathcal{D}}$ is an independent set in $\Gamma_{v, k}$, then it follows from Theorem 9 that $\mathcal{W}_{\Gamma^{\mathcal{D}}}=\mathcal{B}_{\mathcal{D}}$. In particular, we obtain the following corollary.

Corollary 10. If $\mathcal{D}$ is an $S(t, k, v)$ with $t<k$, then the $\mathscr{P}$-position set of $\Gamma^{\mathcal{D}}$ equals $\mathcal{B}_{\mathcal{D}}$.

Example 11. Let $\mathcal{D}$ be the $S(1,2,4)$ with block set $\{\{0,2\},\{1,3\}\}$. As we have seen in the introduction, $\mathcal{P}^{\mathcal{D}}=\left(\begin{array}{c}{[4]} \\ 2\end{array}\right) \backslash\{\{0,1\}\}$ and $\mathcal{W}_{\Gamma^{\mathcal{D}}}=\{\{0,2\},\{1,3\}\}$.

Example 12. The game $\Gamma^{\mathcal{D}_{\text {sh }}}$ equals Hxd. To verify this, it suffices to show that $\mathcal{P}^{\mathcal{D}_{\text {sh }}}=$ $\mathcal{P}_{\text {Hxd }}$. Since $\mathcal{W}_{\text {Hxd }}$ equals the block set $\mathcal{B}_{\text {sh }}$ of $\mathcal{D}_{\text {sh }}$, it follows from Theorem 9 that $\mathcal{P}_{\text {Hxd }} \subseteq$ $\mathcal{P}^{\mathcal{D}_{\text {sh }}}$. We show that $\mathcal{P}^{\mathcal{D}_{\text {sh }}} \subseteq \mathcal{P}_{\mathrm{Hxd}}$. If $B \in \mathcal{B}_{\mathrm{sh}}\left(=\mathcal{W}_{\mathrm{Hxd}}\right)$, then $B \in \mathcal{P}_{\mathrm{Hxd}}$, so $\sum_{b \in B} b \geqslant 21$. This implies that if $P \in \mathcal{P}^{\mathcal{D}_{\text {sh }}}$, then $\sum_{p \in P} p \geqslant 21$. Thus $\mathcal{P}^{\mathcal{D}_{\text {sh }}} \subseteq \mathcal{P}_{\mathrm{Hxd}}$. Therefore $\mathcal{P}^{\mathcal{D}_{\text {sh }}}=$ $\mathcal{P}_{\mathrm{Hxd}}$.

Remark 13. In this paper, we will investigate an $S(t, t+1, v)$ by using the above games obtained from Welter's game. Incidentally, we can also consider games obtained from other than Welter's game to study $S(t, t+m, v)$, especially when $m$ is greater than one. ${ }^{4}$ For example, let $k=t+m$ and let $\Gamma_{v, k}^{m}$ be the game with position set $\left(\begin{array}{c}{[v]} \\ k\end{array}\right)$ and edge set

$$
\left\{(P, Q) \in\left(\begin{array}{c}
{[v]} \\
k
\end{array}\right)^{2}: P \neq Q \text {, there is a walk from } P \text { to } Q \text { of length at most } m \text { in } \Gamma_{v, k}\right\} \text {. }
$$

For example, $(\{1,4,5\},\{0,2,5\})$ is an edge in $\Gamma_{6,3}^{2}$ since $(\{1,4,5\},\{0,4,5\},\{0,2,5\})$ is a walk of length 2 in $\Gamma_{6,3}$. Note that $\Gamma_{v, k}^{1}$ is Welter's game $\Gamma_{v, k}$. Let $\Gamma=\Gamma_{v, k}^{m}$ and let $\mathcal{D}$ be an $S(t, k, v)$ with block set $\mathcal{B}$. Then we can see that $\mathcal{B}$ is an independent set in $\Gamma$. Therefore the $\mathscr{P}$-position set of $\operatorname{Ind}\left(\Gamma, \mathcal{P}_{\Gamma}^{\mathcal{B}}\right)$ is equal to $\mathcal{B}$.

\section{Game Distributions}

We introduce the game distribution of a Steiner system in this section. The Steiner system $\mathcal{D}_{\text {sh }}$ will be characterized by using its game distribution. Hereafter, for $P \in\left(\begin{array}{c}{[v]} \\ k\end{array}\right)$, let

$$
\mathcal{J}(P)=\mathcal{J}_{\Gamma_{v, k}}(P) \text { and } \mathcal{O}(P)=\mathcal{O}_{\Gamma_{v, k}}(P) .
$$

For a $t-(v, k, \lambda)$ design $\mathcal{D}$ and a permutation $\pi$ in $\operatorname{Sym}([v])$, let $\mathcal{D}^{\pi}$ denote the design obtained from $\mathcal{D}$ by applying $\pi$, that is, $\mathcal{D}^{\pi}=\left([v], \mathcal{B}_{\mathcal{D}}^{\pi}\right)$, where $\mathcal{B}_{\mathcal{D}}^{\pi}=\left\{B^{\pi}: B \in \mathcal{B}_{\mathcal{D}}\right\}$ and $B^{\pi}=\left\{b^{\pi}: b \in B\right\}$. Define

$$
\operatorname{Orb}(\mathcal{D})=\left\{\mathcal{D}^{\pi}: \pi \in \operatorname{Sym}([v])\right\}
$$

${ }^{4}$ If $\mathcal{D}$ is an $S(t, t+m, v)$ with $m>1$, then we can show that the number of positions in $\Gamma^{\mathcal{D}}$ always equals $\lambda_{0}+\left(\lambda_{0} k(v-k) / 2\right)$ by Lemma 24 . Thus to investigate $\mathcal{D}$ by using its game distribution, we have to consider other games, for example, $\operatorname{Ind}\left(\Gamma, \mathcal{P}_{\Gamma}^{\mathcal{B}}\right)$ described in Remark 13. 
For example, if $\mathcal{D}=([4],\{\{0,2\},\{1,3\}\})$, then

$$
\begin{aligned}
\operatorname{Orb}(\mathcal{D}) & =\left\{\mathcal{D}, \mathcal{D}^{(12)}, \mathcal{D}^{(23)}\right\} \\
& =\{([4],\{\{0,2\},\{1,3\}\}),([4],\{\{0,1\},\{2,3\}\}),([4],\{\{0,3\},\{1,2\}\})\} .
\end{aligned}
$$

Note that

$$
|\operatorname{Orb}(\mathcal{D})|=\frac{|\operatorname{Sym}([v])|}{|\operatorname{Aut}(\mathcal{D})|}=\frac{v !}{|\operatorname{Aut}(\mathcal{D})|}
$$

Let $f_{\mathcal{D}}(n)$ denote the number of designs $\mathcal{D}^{\prime} \in \operatorname{Orb}(\mathcal{D})$ such that the corresponding game $\Gamma^{\mathcal{D}^{\prime}}$ has $n$ positions, that is,

$$
f_{\mathcal{D}}(n)=\left|\left\{\mathcal{D}^{\prime} \in \operatorname{Orb}(\mathcal{D}):\left|\mathcal{P}^{\mathcal{D}^{\prime}}\right|=n\right\}\right| \text {. }
$$

The function $f_{\mathcal{D}}$ will be called the game distribution of $\mathcal{D}$ (with respect to $\Gamma_{v, k}$ ).

Example 14. Let $\mathcal{D}$ be an $S(1,2,4)$. As we have seen in the introduction, the game distribution of $\mathcal{D}$ is as follows:

$$
\begin{array}{c|ccc|c}
n & 4 & 5 & 6 & \text { Total } \\
\hline f_{\mathcal{D}}(n) & 1 & 1 & 1 & 3
\end{array}
$$

Example 15. Let $\mathcal{D}$ be an $S(2,3,7)$. Then the game distribution of $\mathcal{D}$ is as follows:

\begin{tabular}{c|cccccccc|c}
$n$ & 28 & 29 & 30 & 31 & 32 & 33 & 34 & 35 & Total \\
\hline$f_{\mathcal{D}}(n)$ & 1 & 3 & 5 & 6 & 6 & 5 & 3 & 1 & 30
\end{tabular}

Note that this distribution is symmetric, that is, $f_{\mathcal{D}}(n)=f_{\mathcal{D}}(63-n)$. Incidentally, the game distribution of a Steiner system is not always symmetric. For example, if $\mathcal{D}$ is an $S(2,3,9)$, then its game distribution is as follows:

$$
\begin{array}{c|ccccccccccccc|c}
n & 68 & 69 & 70 & 71 & 72 & 73 & 74 & 75 & 76 & 77 & 78 & 79 & 80 & \text { Total } \\
\hline f_{\mathcal{D}}(n) & 1 & 6 & 16 & 36 & 77 & 94 & 116 & 129 & 131 & 104 & 74 & 39 & 17 & 840
\end{array}
$$

Theorem 16. Let $\mathcal{D}$ be an $S(5,6,12)$. The game distribution of $\mathcal{D}$ is as follows:

$$
\begin{array}{c|cccccccccccc|c}
n & 905 & 906 & 907 & 908 & 909 & 910 & 911 & 912 & 913 & 914 & 915 & 916 & \text { Total } \\
\hline f_{\mathcal{D}}(n) & 1 & 10 & 42 & 150 & 351 & 650 & 1012 & 1237 & 939 & 532 & 115 & 1 & 5040
\end{array}
$$

Moreover, $\Gamma^{\mathcal{D}_{\text {sh }}}$ is the hexad game and is the unique minimum game, that is, $\left|\mathcal{P}^{\mathcal{D}_{s h}}\right|=905$.

Theorem 16 can be obtained by computer.

Remark 17 . Let $\mathcal{D}$ be the unique $S(5,6,12)$ with $\left|\mathcal{P}^{\mathcal{D}}\right|=916$. Then

$$
\begin{array}{r}
\left(\begin{array}{c}
{[12]} \\
6
\end{array}\right) \backslash \mathcal{P}^{\mathcal{D}}=\{ \\
\{0,1,2,3,8,9\},\{0,1,2,4,7,9\},\{0,1,2,4,7,10\},\{0,1,2,5,7,8\}, \\
\{0,1,3,5,6,8\},\{0,1,3,5,7,9\},\{0,1,4,5,6,7\},\{0,2,3,5,6,8\}\}
\end{array}
$$

and the block set $\mathcal{B}_{\mathcal{D}}$ can be obtained from the following MINIMOG: 


\begin{tabular}{c|cccc|}
\hline & 5 & 2 & 6 & 9 \\
+ & 4 & 1 & 11 & 8 \\
- & 3 & 0 & 10 & 7 \\
\hline
\end{tabular}

Remark 18. We close this section by remarking that the game distribution of $S(1,2,2 v)$ can be calculated as follows. If $\mathcal{D}$ is an $S(1,2,2 v)$, then

$$
\sum_{n=0}^{\infty} f_{\mathcal{D}}(n) x^{n}=x^{v^{2}} \prod_{i=1}^{v} \frac{x^{2 i-1}-1}{x-1} .
$$

For example, if $\mathcal{D}$ is an $S(1,2,6)$, then its game distribution and $\sum f_{\mathcal{D}}(n) x^{n}$ are as follows:

$$
\begin{array}{c|ccccccc|c}
n & 9 & 10 & 11 & 12 & 13 & 14 & 15 & \text { Total } \\
\hline f_{\mathcal{D}}(n) & 1 & 2 & 3 & 3 & 3 & 2 & 1 & 15 \\
\sum_{n=0}^{\infty} f_{\mathcal{D}}(n) x^{n}= & x^{9}\left(x^{2}+x+1\right)\left(x^{4}+x^{3}+x^{2}+x+1\right) .
\end{array}
$$

We sketch the proof. Let $\overline{f_{\mathcal{D}}}(n)$ denote the number of designs $\mathcal{D}^{\prime} \in \operatorname{Orb}(\mathcal{D})$ such that the corresponding game $\Gamma^{\mathcal{D}^{\prime}}$ has $n$ non-positions, that is,

$$
\overline{f_{\mathcal{D}}}(n)=\left|\left\{\mathcal{D}^{\prime} \in \operatorname{Orb}(\mathcal{D}):\left|\overline{\mathcal{P}^{\prime}}\right|=n\right\}\right|
$$

where $\overline{\mathcal{P}^{\mathcal{D}}}=\left(\begin{array}{c}{[2 v]} \\ 2\end{array}\right) \backslash \mathcal{P}^{\mathcal{D}}$. Since $\left|\overline{\mathcal{P}^{\mathcal{D}}}\right|+\left|\mathcal{P}^{\mathcal{D}}\right|=\left(\begin{array}{c}2 v \\ 2\end{array}\right)=v(2 v-1)$, it follows that $\overline{f_{\mathcal{D}}}(n)=$ $f_{\mathcal{D}}(v(2 v-1)-n)$. We first show that

$$
\sum_{n=0}^{\infty} \overline{f_{\mathcal{D}}}(n) x^{n}=\prod_{i=1}^{v} \frac{x^{2 i-1}-1}{x-1} .
$$

Let $\Psi_{v}(x)$ be the left-hand side of (4.2). We show (4.2) by induction on $v$. If $v=1$, then $\overline{f_{\mathcal{D}}}(0)=1$ and $\overline{f_{\mathcal{D}}}(n)=0$ for $n \geqslant 1$, so (4.2) holds. Suppose that $v \geqslant 2$, and let $\{0, w\} \in \mathcal{B}_{\mathcal{D}}$. We calculate $\left|\overline{\mathcal{P}^{\mathcal{D}}}\right|$. The number of $P \in \overline{\mathcal{P}^{\mathcal{D}}}$ with $P \cap\{0, w\} \neq \emptyset$ equals $w-1$ since the set of such $P$ is $\{\{0,1\}, \ldots,\{0, w-1\}\}$. Moreover, the number of $P \in \overline{\mathcal{P}^{\mathcal{D}}}$ with $P \cap\{0, w\}=\emptyset$ (i.e., $P \subseteq[2 v] \backslash\{0, w\})$ equals $|\overline{\mathcal{P} \varepsilon}|$, where $\mathcal{E}$ is the $S(1,2,2 v-2)$ described below. Write $[2 v] \backslash\{0, w\}=\left\{a_{0}, \ldots, a_{2 v-3}\right\}$ with $a_{0}<\cdots<a_{2 v-3}$, and let $\phi$ be the bijection $[2 v] \backslash\{0, w\} \ni a_{i} \mapsto i \in[2 v-2]$. Note that $\phi$ is order-preserving, that is, $\phi(a)<\phi(b)$ if and only if $a<b$. Let $\mathcal{E}=\left([2 v-2],\left\{\phi(B): B \in \mathcal{B}_{\mathcal{D}}, B \neq\{0, w\}\right\}\right)$. Then $\mathcal{E}$ is an $S(1,2,2 v-2)$. If $Q \subseteq[2 v] \backslash\{0, w\}$, then $Q \in \overline{\mathcal{P}^{\mathcal{D}}}$ if and only if $\phi(Q) \in \overline{\mathcal{P} \varepsilon}$ since $\phi$ is order-preserving. Therefore the number of $P \in \overline{\mathcal{P}^{\mathcal{D}}}$ with $P \subseteq[2 v] \backslash\{0, w\}$ is equal to $|\overline{\mathcal{P} \varepsilon}|$. Thus $\left|\overline{\mathcal{P}^{\mathcal{D}}}\right|=w-1+|\overline{\mathcal{P} \varepsilon}|$. It follows from the induction hypothesis that

$$
\Psi_{v}(x)=\left(1+x+\cdots+x^{2 v-2}\right) \Psi_{v-1}(x)=\prod_{i=1}^{v} \frac{x^{2 i-1}-1}{x-1} .
$$


Therefore (4.2) holds. Since $f_{\mathcal{D}}(n)=0$ for $n>v(2 v-1)$ and $f_{\mathcal{D}}(n)=\overline{f_{\mathcal{D}}}(v(2 v-1)-n)$, we see that

$$
\begin{aligned}
\sum_{n=0}^{\infty} f_{\mathcal{D}}(n) x^{n} & =\sum_{n=0}^{v(2 v-1)} \overline{f_{\mathcal{D}}}(v(2 v-1)-n) x^{n} \\
& =\sum_{n=0}^{v(2 v-1)} \overline{f_{\mathcal{D}}}(n) x^{v(2 v-1)-n} \\
& =x^{v(2 v-1)} \prod_{i=1}^{v}\left(1+x^{-1}+\cdots+x^{-2 i+2}\right) \\
& =x^{v^{2}} \prod_{i=1}^{v} \frac{x^{2 i-1}-1}{x-1} .
\end{aligned}
$$

\section{$5 \quad$ Symmetric Components}

We show that the game distribution of an $S(t, t+1, v)$ can be decomposed into symmetric components. Using symmetric components, we characterize projective Steiner triple systems in Section 6.

For a $t$ - $(v, k, \lambda)$ design $\mathcal{D}$ and a non-block $P \in\left(\begin{array}{c}{[v]} \\ k\end{array}\right) \backslash \mathcal{B}_{\mathcal{D}}$, a block $B \in \mathcal{B}_{\mathcal{D}}$ is called an out-block of $P$ if $B \in \mathcal{O}(P)$. Define $a_{i}(\mathcal{D})$ to be the number of non-blocks with $i$ out-blocks, that is,

$$
a_{i}(\mathcal{D})=\left|\left\{P \in\left(\begin{array}{c}
{[v]} \\
k
\end{array}\right) \backslash \mathcal{B}_{\mathcal{D}}:\left|\mathcal{O}(P) \cap \mathcal{B}_{\mathcal{D}}\right|=i\right\}\right| .
$$

For example, if $\mathcal{D}=([4],\{\{0,2\},\{1,3\}\})$, then $\{0,1\}$ has no out-blocks; $\{0,3\}$ and $\{1,2\}$ have one out-block, $\{0,2\} ;\{2,3\}$ has two out-blocks, $\{0,2\}$ and $\{1,3\}$; hence

$$
a_{0}(\mathcal{D})=|\{\{0,1\}\}|=1, a_{1}(\mathcal{D})=|\{\{0,3\},\{1,2\}\}|=2, a_{2}(\mathcal{D})=|\{\{2,3\}\}|=1 .
$$

Note that $\left|\mathcal{P}^{\mathcal{D}}\right|=\left|\mathcal{B}_{\mathcal{D}}\right|+\sum_{i \geqslant 1} a_{i}(\mathcal{D})$ and $\left|\overline{\mathcal{P}^{\mathcal{D}}}\right|=a_{0}(\mathcal{D})$, where $\overline{\mathcal{P}^{\mathcal{D}}}=\left(\begin{array}{c}{[v]} \\ k\end{array}\right) \backslash \mathcal{P}^{\mathcal{D}}$. Moreover, if $\mathcal{B}_{\mathcal{D}}$ is an independent set in $\Gamma_{v, k}$, then $a_{i}(\mathcal{D})=0$ for $i \geqslant k+1$. Indeed, if $\left|\mathcal{O}(P) \cap \mathcal{B}_{\mathcal{D}}\right| \geqslant k+1$ for some $P \in\left(\begin{array}{c}{[v]} \\ k\end{array}\right) \backslash \mathcal{B}_{\mathcal{D}}$, then $P^{(p q)}, P^{\left(p q^{\prime}\right)} \in \mathcal{B}_{\mathcal{D}}$ for some $p \in P$ and some $q, q^{\prime} \notin P$ with $q^{\prime}<q$, so $\mathcal{B}_{\mathcal{D}}$ is not an independent set because $\left(P^{(p q)}, P^{\left(p q^{\prime}\right)}\right)$ is an edge of $\Gamma_{v, k}$. Let

$$
s(\mathcal{D})=\left\{a_{0}\left(\mathcal{D}^{\prime}\right)+a_{k}\left(\mathcal{D}^{\prime}\right): \mathcal{D}^{\prime} \in \operatorname{Orb}(\mathcal{D})\right\}
$$

and

$$
\operatorname{Orb}^{\alpha}(\mathcal{D})=\left\{\mathcal{D}^{\prime} \in \operatorname{Orb}(\mathcal{D}): a_{0}\left(\mathcal{D}^{\prime}\right)+a_{k}\left(\mathcal{D}^{\prime}\right)=\alpha\right\}
$$

for $\alpha \in s(\mathcal{D})$. Define

$$
f_{\mathcal{D}}^{\alpha}(n)=\left|\left\{\mathcal{D}^{\prime} \in \operatorname{Orb}^{\alpha}(\mathcal{D}):\left|\mathcal{P}^{\mathcal{D}^{\prime}}\right|=n\right\}\right|
$$


and

$$
\begin{aligned}
\overline{f_{\mathcal{D}}^{\alpha}}(n) & =\left|\left\{\mathcal{D}^{\prime} \in \operatorname{Orb}^{\alpha}(\mathcal{D}):\left|\overline{\mathcal{P}^{\mathcal{D}^{\prime}}}\right|=n\right\}\right| \\
& =\left|\left\{\mathcal{D}^{\prime} \in \operatorname{Orb}^{\alpha}(\mathcal{D}): a_{0}\left(\mathcal{D}^{\prime}\right)=n\right\}\right| .
\end{aligned}
$$

By definition,

$$
f_{\mathcal{D}}(n)=\sum_{\alpha \in s(\mathcal{D})} f_{\mathcal{D}}^{\alpha}(n) .
$$

Let $\mathcal{D}$ be an $S(t, t+1, v)$. Then the following proposition says that $f_{\mathcal{D}}^{\alpha}(n)$ is symmetric. From this, the function $f_{\mathcal{D}}^{\alpha}$ is called a symmetric component of the game distribution of $\mathcal{D}$.

Proposition 19. If $\mathcal{D}$ is an $S(t, k, v)$ with $k=t+1$, and if $\alpha \in s(\mathcal{D})$, then

$$
\overline{f_{\mathcal{D}}^{\alpha}}(n)=\overline{f_{\mathcal{D}}^{\alpha}}(\alpha-n)
$$

for $n \in \mathbb{N}$; in particular,

$$
f_{\mathcal{D}}^{\alpha}(n)=f_{\mathcal{D}}^{\alpha}\left(2\left(\begin{array}{l}
v \\
k
\end{array}\right)-\alpha-n\right)
$$

Proof. It suffices to show the assertion for $\alpha=a_{0}(\mathcal{D})+a_{k}(\mathcal{D})$, since $\overline{f_{\mathcal{D}}^{\alpha}}(n)=\overline{f_{\mathcal{D}^{\prime}}^{\alpha}}(n)$ for $\mathcal{D}^{\prime} \in \operatorname{Orb}(\mathcal{D})$. Let $\mathcal{B}=\mathcal{B}_{\mathcal{D}}$ and $P \in\left(\begin{array}{c}{[v]} \\ k\end{array}\right) \backslash \mathcal{B}$. We first show that

$$
|\mathcal{O}(P) \cap \mathcal{B}|+|\mathcal{J}(P) \cap \mathcal{B}|=k .
$$

Let $p \in P$. Since $k=t+1$, there is a unique $p^{\prime} \in[v]$ such that $P^{\left(p p^{\prime}\right)} \in \mathcal{B}$. Note that $|\mathcal{O}(P) \cap \mathcal{B}|$ equals the number of $p \in P$ with $p^{\prime}<p$. Similarly, $|\mathcal{J}(P) \cap \mathcal{B}|$ equals the number of $p \in P$ with $p^{\prime}>p$. Hence (5.1) holds.

Let $\pi$ be the permutation $i \mapsto v-1-i$ of $[v]$. We next show that $\mathcal{D}^{\pi} \in \operatorname{Orb}^{\alpha}(\mathcal{D})$. Since $p<q \Longleftrightarrow p^{\pi}>q^{\pi}$ for $p, q \in[v]$, it follows that $|\mathcal{J}(P) \cap \mathcal{B}|=\left|\mathcal{O}\left(P^{\pi}\right) \cap \mathcal{B}^{\pi}\right|$. By $(5.1)$,

$$
|\mathcal{O}(P) \cap \mathcal{B}|=i \Longleftrightarrow\left|\mathcal{O}\left(P^{\pi}\right) \cap \mathcal{B}^{\pi}\right|=k-i
$$

This implies that $a_{i}(\mathcal{D})=a_{k-i}\left(\mathcal{D}^{\pi}\right)$, and hence that $a_{0}\left(\mathcal{D}^{\pi}\right)+a_{k}\left(\mathcal{D}^{\pi}\right)=a_{k}(\mathcal{D})+a_{0}(\mathcal{D})=\alpha$. Thus $\mathcal{D}^{\pi} \in \operatorname{Orb}^{\alpha}(\mathcal{D})$. We also see that

$$
a_{0}(\mathcal{D})=n \Longleftrightarrow a_{0}\left(\mathcal{D}^{\pi}\right)=\alpha-n
$$

Therefore $\overline{f_{\mathcal{D}}^{\alpha}}(n)=\overline{f_{\mathcal{D}}^{\alpha}}(\alpha-n)$.

Example 20. The symmetric components of the game distribution of an $S(2,3,9)$ are as shown in Table 1. 
Table 1: The symmetric components of an $S(2,3,9)$.

\begin{tabular}{|c|c|c|c|c|c|c|c|c|c|c|c|c|}
\hline$n$ & $\mid 68$ & 69 & 70 & 71 & 72 & 73 & 74 & 75 & 76 & 77 & 787980 & Total \\
\hline$f_{\mathcal{D}}^{24}(n)$ & 1 & 1 & 1 & 1 & 1 & 1 & 1 & 1 & 1 & & & 9 \\
\hline$f_{\mathcal{D}}^{22}(n)$ & & 5 & 6 & 6 & 7 & 6 & 7 & 6 & 6 & 5 & & 54 \\
\hline$f_{\mathcal{D}}^{20}(n)$ & & & 9 & 14 & 24 & 23 & 22 & 23 & 24 & 14 & 9 & 162 \\
\hline$f_{\mathcal{D}}^{18}(n)$ & & & & 15 & 28 & 40 & 49 & 54 & 49 & 40 & 2815 & 318 \\
\hline$f_{\mathcal{D}}^{16}(n)$ & & & & & 17 & 24 & 37 & 45 & 51 & 45 & $3724 \quad 17$ & 297 \\
\hline$f_{\mathcal{D}}(n)$ & 1 & 6 & & 36 & 77 & 94 & 116 & 129 & 131 & 104 & $\begin{array}{lll}74 & 39 & 17\end{array}$ & 840 \\
\hline
\end{tabular}

Example 21. As shown in Table 2, the game distribution of an $S(2,3,7)$ has a unique symmetric component. This property characterizes projective Steiner triple systems as we will see in Theorem 23.

Table 2: The symmetric component of an $S(2,3,7)$.

\begin{tabular}{c|cccccccc|c}
$n$ & 28 & 29 & 30 & 31 & 32 & 33 & 34 & 35 & Total \\
\hline$f_{\mathcal{D}}^{7}(n)$ & 1 & 3 & 5 & 6 & 6 & 5 & 3 & 1 & 30 \\
\hline$f_{\mathcal{D}}(n)$ & 1 & 3 & 5 & 6 & 6 & 5 & 3 & 1 & 30
\end{tabular}

Example 22. Let $\mathcal{D}$ be an $S(5,6,12)$. Then

$$
a_{i}(\mathcal{D})=a_{6-i}(\mathcal{D}),
$$

so the symmetric components of $f_{\mathcal{D}}$ are as shown in Table 3 . We can prove (5.2) as follows. If $B \in \mathcal{B}_{\mathcal{D}}$, then $\bar{B} \in \mathcal{B}_{\mathcal{D}}$, where $\bar{B}=[12] \backslash B$. This implies that if $P \in\left(\begin{array}{c}{[12]} \\ 6\end{array}\right) \backslash \mathcal{B}_{\mathcal{D}}$ and $P^{(p q)} \in \mathcal{B}_{\mathcal{D}}$, then

$$
\bar{P} \in\left(\begin{array}{c}
{[12]} \\
6
\end{array}\right) \backslash \mathcal{B}_{\mathcal{D}} \quad \text { and } \quad \bar{P}^{(p q)}=\overline{P^{(p q)}} \in \mathcal{B}_{\mathcal{D}}
$$

For example, if $\mathcal{D}=\mathcal{D}_{\text {sh }}$ and $P=\{1,2,3,4,5,11\}$, then $P^{(50)}=\{1,2,3,4,0,11\} \in \mathcal{B}_{\mathcal{D}}$ and $\bar{P}^{(50)}=\{0,6,7,8,9,10\}^{(50)}=\{5,6,7,8,9,10\} \in \mathcal{B}_{\mathcal{D}}$. Note that if $P^{(p q)} \in \mathcal{O}(P)$, then $\bar{P}^{(p q)} \in \mathcal{J}(\bar{P})$. It follows that if $\left|\mathcal{O}(P) \cap \mathcal{B}_{\mathcal{D}}\right|=i$, then $\left|\mathcal{O}(\bar{P}) \cap \mathcal{B}_{\mathcal{D}}\right|=6-i$. Hence $a_{i}(\mathcal{D})=a_{6-i}(\mathcal{D})$, so its symmetric components are as shown in Table 3 . The symmetric components of the game distribution of an $S(4,5,11)$ are also shown in Table 4.

Table 3: The symmetric components of an $S(5,6,12)$.




Table 4: The symmetric components of an $S(4,5,11)$.

\begin{tabular}{c|cccccccccccc|c}
$n$ & 443 & 444 & 445 & 446 & 447 & 448 & 449 & 450 & 451 & 452 & 453 & 454 & Total \\
\hline$f_{\mathcal{D}}^{29}(n)$ & 1 & 1 & 3 & & & & & 3 & 1 & 1 & & & 10 \\
$f_{\mathcal{D}}^{28}(n)$ & & 7 & 7 & 12 & 8 & 8 & 8 & 12 & 7 & 7 & & 76 \\
$f_{\mathcal{D}}^{27}(n)$ & & 2 & 25 & 62 & 62 & 77 & 77 & 62 & 62 & 25 & 2 & & 456 \\
$f_{\mathcal{D}}^{26}(n)$ & & & 7 & 44 & 106 & 155 & 212 & 155 & 106 & 44 & 7 & & 836 \\
$f_{\mathcal{D}}^{25}(n)$ & & & & 31 & 167 & 220 & 345 & 345 & 220 & 167 & 31 & & 1526 \\
$f_{\mathcal{D}}^{24}(n)$ & & & & 1 & 8 & 140 & 259 & 402 & 259 & 140 & 8 & 1 & 1218 \\
$f_{\mathcal{D}}^{23}(n)$ & & & & & & 50 & 101 & 216 & 216 & 101 & 50 & & 734 \\
$f_{\mathcal{D}}^{22}(n)$ & & & & & & & 10 & 36 & 58 & 36 & 10 & & 150 \\
$f_{\mathcal{D}}^{21}(n)$ & & & & & & & & 6 & 9 & 9 & 6 & & 30 \\
$f_{\mathcal{D}}^{20}(n)$ & & & & & & & & & 1 & 2 & 1 & & 4 \\
\hline$f_{\mathcal{D}}(n)$ & 1 & 10 & 42 & 150 & 351 & 650 & 1012 & 1237 & 939 & 532 & 115 & 1 & 5040
\end{tabular}

\section{A Characterization of Projective Steiner Triple Systems}

Theorem 23. If $\mathcal{D}$ is an $S(2,3, v)$, then $\mathcal{D}$ is projective if and only if its game distribution has a unique symmetric component, that is, $|s(\mathcal{D})|=1$.

To prove Theorem 23, we show two lemmas.

Let $\mathcal{D}$ be an $S(2,3, v)$. The following lemma enables us to calculate $a_{0}(\mathcal{D})+a_{3}(\mathcal{D})$. Recall that $\lambda_{i}$, defined in (2.1), is the number of blocks of $\mathcal{D}$ containing $i$ given points.

Lemma 24. Let $\mathcal{D}$ be a $t-(v, k, \lambda)$ design with block set $\mathcal{B}$.

(1) If $t \geqslant 1$ and $\mathcal{B}$ is an independent set in $\Gamma_{v, k}$, then

$$
\sum_{i=0}^{k} i a_{i}(\mathcal{D})=\frac{\lambda_{0} k(v-k)}{2} .
$$

(2) If $\mathcal{D}$ is an $S(2,3, v)$, then

$$
a_{0}(\mathcal{D})+a_{3}(\mathcal{D})=\sum_{\{B, C\} \in\left(\begin{array}{l}
\mathcal{B} \\
2
\end{array}\right)} I(B, C)-\frac{v(v-1)(v-3)}{12},
$$

where $I(B, C)=|\mathcal{J}(B) \cap \mathcal{J}(C)|$.

Proof. (1) Let

$$
\mathcal{A}_{i}(\mathcal{D})=\left\{P \in\left(\begin{array}{c}
{[v]} \\
k
\end{array}\right) \backslash \mathcal{B}:|\mathcal{O}(P) \cap \mathcal{B}|=i\right\} .
$$

Then $a_{i}(\mathcal{D})=\left|\mathcal{A}_{i}(\mathcal{D})\right|$. Since $\mathcal{B}$ is an independent set, we see that $\bigcup_{i=0}^{k} \mathcal{A}_{i}(\mathcal{D})=\left(\begin{array}{c}{[v]} \\ k\end{array}\right) \backslash \mathcal{B}$ and

$$
\sum_{i=0}^{k} i a_{i}(\mathcal{D})=\sum_{i=0}^{k}\left|\left\{(P, B): P \in \mathcal{A}_{i}(\mathcal{D}), B \in \mathcal{O}(P) \cap \mathcal{B}\right\}\right|
$$




$$
\begin{aligned}
& =\left|\left\{(P, B): P \in\left(\begin{array}{c}
{[v]} \\
k
\end{array}\right) \backslash \mathcal{B}, B \in \mathcal{O}(P) \cap \mathcal{B}\right\}\right| \\
& =|\{(P, B): B \in \mathcal{B}, P \in \mathcal{J}(B)\}| \\
& =\sum_{B \in \mathcal{B}}|\mathcal{J}(B)| .
\end{aligned}
$$

Let $B \in \mathcal{B}$ and write $B=\left\{b_{1}, \ldots, b_{k}\right\}$ with $b_{1}<\cdots<b_{k}$. Then $P \in \mathcal{J}(B)$ if and only if $P=B^{\left(b_{i} p\right)}$ for some $b_{i}$ and $p \notin B$ with $p>b_{i}$. The number of such $p$ equals $v-1-b_{i}-(k-i)$. Hence

$$
|\mathcal{J}(B)|=\sum_{i=1}^{k}\left(v-1-b_{i}-(k-i)\right)=k(v-1)-\sum_{b \in B} b-\frac{k(k-1)}{2} .
$$

Since

$$
\begin{aligned}
\sum_{B \in \mathcal{B}} \sum_{b \in B} b & =(0+1+\cdots+v-1) \lambda_{1} \\
& =\frac{v(v-1)}{2} \frac{\left(\begin{array}{c}
v-1 \\
t-1
\end{array}\right)}{\left(\begin{array}{c}
k-1 \\
t-1
\end{array}\right)} \lambda \\
& =\frac{k(v-1)}{2} \frac{\left(\begin{array}{l}
v \\
t
\end{array}\right)}{\left(\begin{array}{c}
k \\
t
\end{array}\right)} \lambda \\
& =\frac{k(v-1) \lambda_{0}}{2},
\end{aligned}
$$

it follows that

$$
\sum_{B \in \mathcal{B}}|\mathcal{J}(B)|=\lambda_{0}\left(k(v-1)-\frac{k(v-1)}{2}-\frac{k(k-1)}{2}\right)=\frac{\lambda_{0} k(v-k)}{2} .
$$

(2) Let $a_{i}=a_{i}(\mathcal{D})$. We first show that

$$
\sum_{i=0}^{3}\left(\begin{array}{l}
i \\
2
\end{array}\right) a_{i}=a_{2}+3 a_{3}=\sum_{\{B, C\} \in\left(\begin{array}{c}
\mathcal{B} \\
2
\end{array}\right)} I(B, C) .
$$

By the definition of $a_{i}$,

$$
\begin{aligned}
\sum_{i=0}^{3}\left(\begin{array}{l}
i \\
2
\end{array}\right) a_{i} & =\sum_{i=0}^{3}\left|\left\{(P,\{B, C\}): P \in \mathcal{A}_{i}(\mathcal{D}),\{B, C\} \in\left(\begin{array}{c}
\mathcal{O}(P) \cap \mathcal{B} \\
2
\end{array}\right)\right\}\right| \\
& =\left|\left\{(P,\{B, C\}): P \in\left(\begin{array}{c}
{[v]} \\
3
\end{array}\right) \backslash \mathcal{B},\{B, C\} \in\left(\begin{array}{c}
\mathcal{O}(P) \cap \mathcal{B} \\
2
\end{array}\right)\right\}\right| \\
& =\left|\left\{(P,\{B, C\}):\{B, C\} \in\left(\begin{array}{l}
\mathcal{B} \\
2
\end{array}\right), P \in \mathcal{J}(B) \cap \mathcal{J}(C)\right\}\right|
\end{aligned}
$$




$$
=\sum_{\{B, C\} \in\left(\begin{array}{c}
\mathcal{B} \\
2
\end{array}\right)} I(B, C)
$$

We now calculate $a_{0}+a_{3}$. Note that $\lambda_{0}=v(v-1) / 6$. By $(1)$,

$$
\sum_{i=0}^{3} i a_{i}=a_{1}+2 a_{2}+3 a_{3}=\frac{\lambda_{0} 3(v-3)}{2}=\frac{v(v-1)(v-3)}{4} .
$$

Since

$$
\sum_{i=0}^{3} a_{i}=a_{0}+a_{1}+a_{2}+a_{3}=\left(\begin{array}{l}
v \\
3
\end{array}\right)-\lambda_{0}=\frac{v(v-1)(v-3)}{6}
$$

it follows that

$$
\sum_{i=0}^{3} i a_{i}-\sum_{i=0}^{3} a_{i}=-a_{0}+a_{2}+2 a_{3}=\frac{v(v-1)(v-3)}{12} .
$$

Therefore

$$
a_{0}+a_{3}+\frac{v(v-1)(v-3)}{12}=a_{2}+3 a_{3}=\sum_{\{B, C\} \in\left(\begin{array}{c}
\mathcal{B} \\
2
\end{array}\right)} I(B, C) .
$$

From Lemma 24, to calculate $a_{0}+a_{3}$, it is enough to compute $\sum I(B, C)$. To do this, the following easy lemma will be used.

Lemma 25. Let $B$ and $C$ be two distinct blocks of an $S(2,3, v)$.

(1) If $I(B, C) \neq 0$, then $|B \cap C|=1$.

(2) If $B=\left\{x, b, b^{\prime}\right\}, C^{\prime}=\left\{x, c, c^{\prime}\right\}, b<b^{\prime}$, and $b<c<c^{\prime}$, then

$$
I(B, C)=\left[c<b^{\prime}\right]+\left[c^{\prime}<b^{\prime}\right]
$$

where [ ] is the Iverson bracket notation, that is, $[S]=1$ if a statement $S$ holds, and $[S]=0$ otherwise.

(3) If

$$
I(B, C) \neq I\left(B^{(i i+1)}, C^{(i i+1)}\right),
$$

then $\{B, C\}=\{\{i, x, y\},\{i+1, x, z\}\}$ for some $x, y, z \in[v]$ with $y \neq z$.

Proof. (1) If $B \cap C=\emptyset$, then $I(B, C)=0$. If $|B \cap C| \geqslant 2$, then $B=C$. Hence $|B \cap C|=1$. (2) Since $b<b^{\prime}$ and $b<c<c^{\prime}$, it follows that

$$
\mathcal{J}(B) \cap \mathcal{J}(C)= \begin{cases}\emptyset & \text { if } b^{\prime}<c, \\ \left\{\left\{x, b^{\prime}, c^{\prime}\right\}\right\} & \text { if } c<b^{\prime}<c^{\prime}, \\ \left\{\left\{x, b^{\prime}, c^{\prime}\right\},\left\{x, b^{\prime}, c\right\}\right\} & \text { if } c^{\prime}<b^{\prime} .\end{cases}
$$


Thus (6.2) holds.

(3) By (1), if $|B \cap C| \neq 1$ then $I(B, C)=I\left(B^{(i i+1)}, C^{(i i+1)}\right)$. Let $B=\left\{x, b, b^{\prime}\right\}$ and $C=\left\{x, c, c^{\prime}\right\}$. We may assume that $b<b^{\prime}$ and $b<c<c^{\prime}$. If

$$
\left[y^{(i i+1)}<z^{(i i+1)}\right] \neq[y<z]
$$

then $\{y, z\}=\{i, i+1\}$. It follows from (2) that $\{i, i+1\} \in\left\{\{b, c\},\left\{b^{\prime}, c\right\},\left\{b^{\prime}, c^{\prime}\right\}\right\}$. Hence $\{B, C\}=\{\{x, i, y\},\{x, i+1, z\}\}$ for some $y, z \in[v]$ with $y \neq z$.

Proof of Theorem 23. Let $\mathcal{D}$ be an $S(2,3, v)$ with block set $\mathcal{B}$.

We first show that if $\mathcal{D}$ is projective, then $|s(\mathcal{D})|=1$. By Lemma 25,

$$
\sum_{\{B, C\} \in\left(\begin{array}{c}
\mathcal{B} \\
2
\end{array}\right)} I(B, C)=\sum_{\{B, C\} \in\left(\begin{array}{c}
\mathcal{B} \\
2
\end{array}\right),|B \cap C|=1} I(B, C) .
$$

Let $\left\{x, b, b^{\prime}\right\}$ and $\left\{x, c, c^{\prime}\right\}$ be two distinct blocks of $\mathcal{D}$. We may assume that $b<b^{\prime}$ and $b<c<c^{\prime}$. Since $\mathcal{D}$ is projective, there are $y, z \in[v]$ such that $\{y, b, c\},\left\{y, b^{\prime}, c^{\prime}\right\},\left\{z, b, c^{\prime}\right\}$, and $\left\{z, b^{\prime}, c\right\}$ are blocks. Thus we obtain the following triple of pairs of blocks:

$$
\left\{\left\{\left\{x, b, b^{\prime}\right\},\left\{x, c, c^{\prime}\right\}\right\}, \quad\left\{\{y, b, c\},\left\{y, b^{\prime}, c^{\prime}\right\}\right\}, \quad\left\{\left\{z, b, c^{\prime}\right\},\left\{z, b^{\prime}, c\right\}\right\}\right\} .
$$

By Lemma 25,

$$
\begin{aligned}
I\left(\left\{x, b, b^{\prime}\right\}\right. & \left.,\left\{x, c, c^{\prime}\right\}\right)+I\left(\{y, b, c\},\left\{y, b^{\prime}, c^{\prime}\right\}\right)+I\left(\left\{z, b, c^{\prime}\right\},\left\{z, b^{\prime}, c\right\}\right) \\
& =\left[c<b^{\prime}\right]+\left[c^{\prime}<b^{\prime}\right]+\left[b^{\prime}<c\right]+\left[c^{\prime}<c\right]+\left[b^{\prime}<c^{\prime}\right]+\left[c<c^{\prime}\right]=3 .
\end{aligned}
$$

Note that every pair $\{B, C\}$ of blocks with $|B \cap C|=1$ belongs to exactly one such triple and there are $v\left(\begin{array}{c}\lambda_{1} \\ 2\end{array}\right) / 3$ triples. Hence

$$
\sum_{\{B, C\} \in\left(\begin{array}{c}
B \\
2
\end{array}\right),|B \cap C|=1} I(B, C)=\frac{v\left(\begin{array}{c}
\lambda_{1} \\
2
\end{array}\right)}{3} 3=\frac{v(v-1)(v-3)}{8} .
$$

By Lemma 24,

$$
a_{0}(\mathcal{D})+a_{3}(\mathcal{D})=\sum_{\{B, C\} \in\left(\begin{array}{c}
\mathcal{B} \\
2
\end{array}\right)} I(B, C)-\frac{v(v-1)(v-3)}{12}=\frac{v(v-1)(v-3)}{24} .
$$

Therefore $s(\mathcal{D})=\{v(v-1)(v-3) / 24\}$.

We next show that if $\mathcal{D}$ is not projective, then $|s(\mathcal{D})|>1$. Note that $v \geqslant 9$. Since $\mathcal{D}$ is not projective, it follows from Remark 4 that we may assume that

$$
\{0, v-2,2\},\{1, v-2,3\},\{0, v-3,3\},\{1, v-3,4\} \in \mathcal{B}
$$

by replacing $\mathcal{D}$ with some $\mathcal{D}^{\prime} \in \operatorname{Orb}(\mathcal{D})$ if necessary (see Figure 7 ). We may also assume 


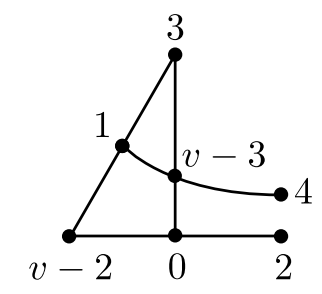

Figure 7: The four blocks of $\mathcal{D}$.

that $\{0,1, v-1\} \in \mathcal{B}$. We show that



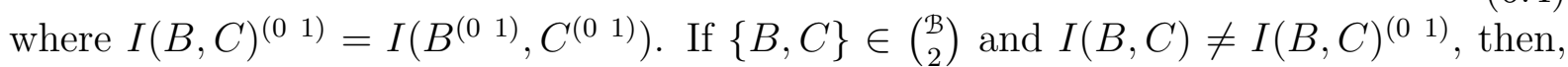
by Lemma 25 ,

$$
\{B, C\}=\{\{0, x, y\},\{1, x, z\}\}
$$

for some $x, y, z \in[v]$ with $y \neq z$; note that $x \neq v-1$ since $\{0,1, v-1\} \in \mathcal{B}$. For $x \in\{2,3, \ldots, v-2\}$, let $B_{x}=\left\{0, x, y_{x}\right\} \in \mathcal{B}$ and $C_{x}=\left\{1, x, z_{x}\right\} \in \mathcal{B}$. By assumption,

$$
\begin{aligned}
& B_{v-2}=\{0, v-2,2\}, C_{v-2}=\{1, v-2,3\}, \\
& B_{v-3}=\{0, v-3,3\}, C_{v-3}=\{1, v-3,4\} .
\end{aligned}
$$

We see that

$$
\sum_{\{B, C\} \in\left(\begin{array}{c}
\mathcal{B} \\
2
\end{array}\right)} I(B, C)-I(B, C)^{(01)}=\sum_{x=2}^{v-2} I\left(B_{x}, C_{x}\right)-I\left(B_{x}, C_{x}\right)^{(01)} .
$$

Similarly, by Lemma 25 , if $\{B, C\} \in\left(\begin{array}{c}\mathcal{B} \\ 2\end{array}\right)$ and $I(B, C)^{(23)} \neq I(B, C)^{(23)(01)}$, then

$$
\left\{B^{(23)}, C^{(23)}\right\}=\left\{\{0, x, y\}^{(23)},\{1, x, z\}^{(23)}\right\}
$$

for some $x, y, z \in[v]$ with $y \neq z$, and hence $\{B, C\}=\left\{B_{x}, C_{x}\right\}$. Therefore

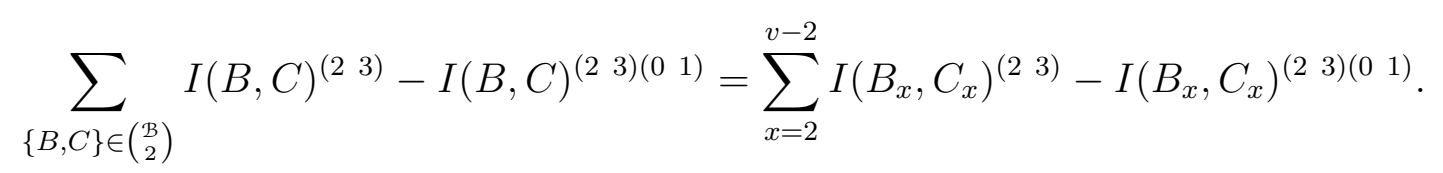

If $I\left(B_{x}, C_{x}\right) \neq I\left(B_{x}, C_{x}\right)^{(23)}$, then $\left\{B_{x}, C_{x}\right\}=\{\{2, x, u\},\{3, x, w\}\}$ for some $u, w \in[v]$, so $x=v-2$ by (6.5). Similarly, if $I\left(B_{x}, C_{x}\right)^{(01)} \neq I\left(B_{x}, C_{x}\right)^{(23)(01)}$, then $x=v-2$. Let $B_{o}=B_{v-2}$ and $C_{o}=C_{v-2}$. By (6.6) and (6.7),

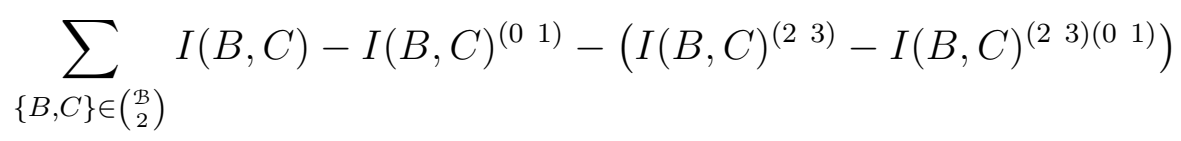




$$
\begin{aligned}
& \left.=\sum_{x=2}^{v-2} I\left(B_{x}, C_{x}\right)-I\left(B_{x}, C_{x}\right)^{(0} 1\right)-\left(I\left(B_{x}, C_{x}\right)^{(23)}-I\left(B_{x}, C_{x}\right)^{\left(\begin{array}{lll}
2 & 3
\end{array}\right)\left(\begin{array}{ll}
0 & 1
\end{array}\right)}\right)
\end{aligned}
$$

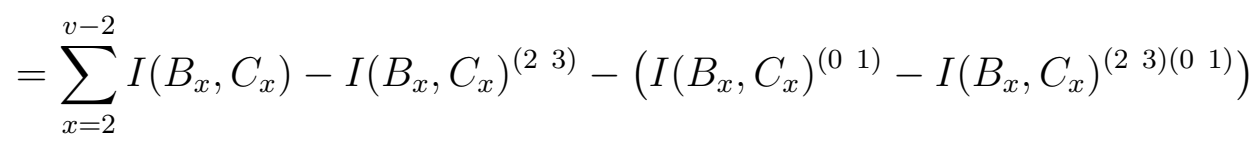

$$
\begin{aligned}
& =I\left(B_{o}, C_{o}\right)-I\left(B_{o}, C_{o}\right)^{(23)}-\left(I\left(B_{o}, C_{o}\right)^{\left(\begin{array}{ll}
2 & 1
\end{array}\right)}-I\left(B_{o}, C_{o}\right)^{\left(\begin{array}{lll}
2 & 3
\end{array}\right)\left(\begin{array}{ll}
0 & 1
\end{array}\right)}\right) .
\end{aligned}
$$

Since

$$
\begin{aligned}
& I\left(B_{o}, C_{o}\right)=I(\{0, v-2,2\},\{1, v-2,3\})=1, \\
& I\left(B_{o}, C_{o}\right)^{(23)}=I(\{0, v-2,3\},\{1, v-2,2\})=2, \\
& I\left(B_{o}, C_{o}\right)^{(01)}=I(\{1, v-2,2\},\{0, v-2,3\})=2, \\
& I\left(B_{o}, C_{o}\right)^{(23)(01)}=I(\{1, v-2,3\},\{0, v-2,2\})=1,
\end{aligned}
$$

we see that

$\sum_{\{B, C\} \in\left(\begin{array}{c}\mathcal{B} \\ 2\end{array}\right)} I(B, C)-I(B, C)^{(01)}-\left(I(B, C)^{(23)}-I(B, C)^{(23)\left(\begin{array}{ll}(2 & 1\end{array}\right)}\right)=1-2-(2-1)=-2$.

Therefore (6.4) holds. It follows from Lemma 24 that $|s(\mathcal{D})|>1$.

\section{Acknowledgments}

The author would like to thank the anonymous referees for their valuable comments.

\section{References}

[1] A. Baartmans, I. Landjev, and V. D. Tonchev. On the binary codes of Steiner triple systems. Des. Codes Cryptogr., 8:29-43, 1996.

[2] E. R. Berlekamp, J. H. Conway, and R. K. Guy. Winning Ways for Your Mathematical Plays, vol. 1. A K Peters, Natick, MA, 2nd ed., 2001.

[3] C. J. Colbourn and A. Rosa. Triple Systems. Clarendon Press, Oxford, 1999.

[4] F. N. Cole, L. D. Cummings, and H. S. White. The complete enumeration of triad systems in 15 elements. Proc. Natl. Acad. Sci. USA, 3(3):197-199, 1917.

[5] J. H. Conway. On Numbers and Games. A K Peters, Natick, MA, 2nd ed., 2001.

[6] J. H. Conway and N. J. A. Sloane. Lexicographic codes: Error-correcting codes from game theory. IEEE Trans. Inform. Theory, 32(3):337-348, 1986.

[7] J. H. Conway and N. J. A. Sloane. Sphere Packings, Lattices and Groups. Springer, New York, NY, 3rd ed., 1999. 
[8] V. de Pasquale. Sui sistemi ternari di 13 elementi. Rendiconti Reale Istituto Lombardo di Scienze e Lettere, 2(32):213-221, 1899.

[9] M. Dehon. Designs et hyperplans. J. Combin. Theory Ser. A, 23(3):264-274, 1977.

[10] P. Diaconis, R. L. Graham, and W. M. Kantor. The mathematics of perfect shuffles. Adv. Appl. Math., 4(2):175-196, 1983.

[11] J. Doyen, X. Hubaut, and M. Vandensavel. Ranks of incidence matrices of Steiner triple systems. Math. Z., 163:251-259, 1978.

[12] A. S. Fraenkel and U. Larsson. Games on arbitrarily large rats and playability. Integers, 19:\#G04, 2019.

[13] J. Kahane and A. J. Ryba. The hexad game. Electron. J. Combin., 8(2):\#R11, 2001.

[14] T. P. Kirkman. On a problem in combinations. Cambridge and Dublin Mathematical Journal, 2:191-204, 1847.

[15] E. H. Moore. Concerning triple systems. Math. Ann., 43:271-285, 1893.

[16] O. Veblen and J. W. Young. Projective Geometry. Ginn and Company, Boston, 1910.

[17] E. Witt. Die 5-fach transitiven gruppen von Mathieu. Abh. Math. Semin. Univ. Hambg., 12:256-264, 1938.

[18] E. Witt. Über Steinersche systeme. Abh. Math. Semin. Univ. Hambg., 12:265-275, 1938. 